which displays increased oxidative stress and triglyceride contents in the skeletal muscle. Experimental evidence suggests that ApoE KO mice have impaired regeneration after injury. Skeletal muscle stem cells are responsible for the regenerative capacity of skeletal muscle and undergo activation, proliferation and differentiation upon injury in order to support muscle repair and regeneration. The skeletal muscle stem cell function of ApoE KO mice remains largely unknown. We hypothesised that the attenuated regenerative capacity of the ApoE-deficient muscle derives from deficits in skeletal muscle stem cell function. Therefore, we investigated the impact of ApoE deficiency on the proliferation and differentiation profiles of muscle stem cells.

Methods Muscle stem cells from the extensor digitorum longus and biceps brachii were isolated from male ApoE KO and wild type mice for single fibre culture experiments ex vivo and stem cell cultures in vitro. Stem cell proliferation and differentiation, morphology, immunohistochemistry and gene expression analysis were performed. Cell culture experiments were conducted with $n=6-9$ technical replicates and $n=2-3$ independent experiments. Significant statistical differences were determined by the Mann-Whitney $U$ test for $\mathrm{p}<0.05$.

Results Single fibres of wild type and ApoE $\mathrm{KO}$ mice did not markedly differ in their myogenic proliferation over the first $48 \mathrm{~h}$ in culture. After 72 hours however, impaired differentiation of ApoE KO mouse muscle stem cells was evident on single fibres ex vivo based on reduced expression of the muscle regulatory factor myogenin. Furthermore, treatment of cultures with $0.5 \mathrm{mM}$ palmitate for $24 \mathrm{~h}$ resulted in loss of stemness and increased commitment to differentiation (i.e. Pax7-ve/ MyoD+ve cells) as well as increased myogenic differentiation. Isolated muscle stem cells from ApoE KO mouse displayed impaired proliferation as well as differentiation, with morphological studies resulting in reduced myotube width and length, an indicator of reduced myotube fusion. These results were exacerbated by application of palmitate in both wild type and ApoE KO. These findings are strengthened by reduced gene expression data for key factors regulating skeletal muscle fusion of myogenic progenitors, cell fate and contractility (i.e. MyoD, Scrib1, myogenin, Bex1, tmem8c, srf, mhc1 and acta1).

Conclusion We report for the first time impaired skeletal muscle stem cell function in hyperlipidaemic ApoE KO mice, which may account for the impaired regenerative capacity of this mouse strain. These data establish a link between muscle stem biology and impaired myogenesis in an experimental model of atherosclerosis and systemic hyperlipidaemia with implications for the myopathies seen in cardiovascular disorders.

Conflict of interest None

\section{BS56 REDOX-REGULATION OF ANGII-INDUCED CEREBRAL MICROVASCULAR DAMAGE IN AGING}

Li Geng*, Jian-Mei Li. University of Reading

10.1136/heartjnl-2019-BCS.217

Oxidative damage of cerebral vasculature has been found to play an important role in aging-related neurodegeneration. Endothelial oxidative stress attributable to the activation of a Nox2-containing NADPH oxidase is an early sign of agerelated vascular abnormalities. However, the mechanism of aging-related Nox2 activation and cerebral microvasculature rarefaction remains unclear. In this study we used littermates of wild-type (WT) and Nox2 knockout (Nox2KO) mice at young $(3-4 \mathrm{~m})$ and old age $(20-22 \mathrm{~m})$ to investigate the role of Nox2-derived ROS in oxidative damage of cerebral microvasculature in aging. Compared to WT young mice, WT (but not the Nox2KO) aging mice had higher blood pressure and elevated serum levels of AngII. WT aging brains had significantly higher levels of AngII, increased ROS production, elevated lipid peroxidation, reduced capillary density and increased expressions of cell apoptosis markers i.e. increased p53 expression and $\gamma \mathrm{H} 2 \mathrm{AX}$ phosphorylation $(\mathrm{p}<0.05)$. However, these AngII-induced abnormalities were significantly reduced or absent in Nox $2 \mathrm{KO}$ aging brains. The mechanism of AngII-induced Nox2 activation and cerebral endothelial oxidative damage was further investigated in vitro using primary cerebral microvascular endothelial cells isolated from mid-aged WT mice. AngII $(100 \mu \mathrm{mol} / \mathrm{L})$ increased significantly the levels of endothelial ROS production, Nox2 expression and p47phox phosphorylation. These were accompanied by increased ERK1/2 and $\gamma \mathrm{H} 2 \mathrm{AX}$ phosphorylation and p53 expression detected by Western Blot and damaged capillary formation on Matri-gels. However, all these AngII-induced oxidative responses were inhibited in the presence of a specific Nox2 inhibitor (Nox2-ds-tat). In conclusion, Nox2derived ROS and redox signaling plays a critical role in regulation of AngII-induced cerebral microvascular rarefaction in aging.

Conflict of interest No

\section{BS57 ABSTRACT WITHDRAWN}

\section{BS58 DOES CHANGE IN RENAL FUNCTION AFFECT WARFARIN'S EFFICACY IN PATIENTS WITH ATRIAL FIBRILLATION?}

${ }^{1}$ Ahsan Khan*, ${ }^{2}$ Eduard Shantsila, ${ }^{2}$ Y.C. Lau, ${ }^{3}$ Lewis Hardy, ${ }^{3}$ Helen Philippou, ${ }^{1}$ Gregory Lip. ${ }^{1}$ Liverpool Heart \& Chest Hospital NHS Foundation Trust; ${ }^{2}$ University of Birmingham; ${ }^{3}$ University of Leeds

\subsection{6/heartjnl-2019-BCS.218}

Introduction Anticoagulation use in atrial fibrillation (AF) is common, but concurrent chronic kidney disease (CKD) reduces its efficacy and potentiates the risk of bleeding. Patients with AF have a higher incidence of renal impairment and the latter predisposes to incident AF. The co-existence of both conditions leads to a higher risk for thromboembolicrelated adverse events but also a paradoxical increased haemorrhagic risk with devastating consequences for the individual. Assessing the effect of renal function on plasma clot properties in anticoagulated AF patients may help explain reasons behind this.

Aim To observe how progressive renal dysfunction affects plasma clot properties in AF patients on warfarin.

Methods We studied 192 patients with AF on warfarin and divided them in 4 groups based on estimated glomerular filtration rate (eGFR), eGFR $>90 \quad(n=30)$, eGFR 60-89 $(n=89)$, eGFR 30-59 $(n=59)$ and eGFR $<30 \quad(n=14)$. Plasma samples were obtained from patients using venepuncture and centrifuged to prepare platelet poor plasma. Assays were performed 